\title{
Role of activated carbon features on the photocatalytic degradation of phenol
}

\author{
Leticia F. Velasco*, José B. Parra, Conchi O. Ania \\ ${ }^{a}$ Instituto Nacional del Carbón, CSIC, Oviedo 33080, Spain
}

*Corresponding author E-mail: leticia@incar.csic.es (LF Velasco)

Tel.: +34985119090; Fax: +34-985297662

\begin{abstract}
In this work we have investigated the role of a porous carbon material used as photocatalyst itself, and catalyst support in a carbon/titania composite towards the photodegradation of phenol, and compared the results to that of bare titanium oxide. The immobilization of titania on an activated carbon provoked an acceleration of the degradation rate under UV irradiation, which is likely attributed to the porosity of the carbon support. The identification of the degradation intermediates detected in the solution showed that the presence of the carbon support affects the nature of phenol degradation mechanism through the formation of different intermediates. Additionally, phenol photodecomposition rate over the carbon support outperformed that attained in the carbon/titania composite, suggesting an important self-photoactivity of the carbon support.
\end{abstract}

\section{Keywords}

photodegradation, phenol, activated carbon, composite

\section{INTRODUCTION}

Hazardous organic compounds are one of the major causes of environmental pollution, particularly in water resources. Photo-catalysis has proven to be a promising technology for the degradation of these compounds, being titania $\left(\mathrm{TiO}_{2}\right)$ the most commonly used photocatalyst because it is nontoxic, photo-stable, cheap and very efficient under ultraviolet light irradiation [1]. Whereas most studies focus on the modification and performance of titania, the use of carbon- $\mathrm{TiO}_{2}$ composites has been scarcely explored for the photocatalytic degradation of toxic pollutants $[2,3]$. In this regard, it has been shown that the mixture activated carbon (AC) and $\mathrm{TiO}_{2}$ can have a synergistic effect for the photodegradation of organic pollutants $[4,5]$. Moreover, the activity of the carbon-titania composites -provided by $\mathrm{TiO}_{2}$ particles- also is expected to have a strong dependency on the textural and chemical composition of the carbon material, being a key factor in the performance of the resulting carbon-titania composite. The porosity of the AC provides a high adsorption of the pollutants on the catalyst surface, and therefore it might accelerate the process of their decomposition through the transfer of the adsorbed molecules to the surface of the photoactive titania. 
The aim of this work was to investigate carbon/ $/ \mathrm{TiO}_{2}$ composites for the photodegradation of phenol in aqueous solutions, and to comprehend the role of the activated carbon properties on the performance of the studied catalysts. As probe molecule we have selected phenol, one of the most refractarious aromatic compounds frequently found in wastewater. The efficiency of the carbontitania catalyst in the photo-assisted degradation of phenol in aqueous solutions was discussed in terms of the chemical and textural properties of the carbon support.

\section{EXPERIMENTAL}

\subsection{MATERIALS}

We have selected a commercial activated carbon obtained from physical activation of bituminous coal -Q- as catalyst support (particle size $0.212-0.710 \mathrm{~mm}$ ). This carbon is characterized by a low oxygen content (ca. 2.1 wt.\%) and a basic nature (point of zero charge of $8.9 \mathrm{pH}$ units). The titania-carbon composite was prepared by infiltration of a suspension in ethanol of commercial titanium oxide (P25 Degussa) on the activated carbon (ratio carbon: titania 85:15) in a rotary evaporator under vacuum for 2 hour. After the rotation, the solvent (ethanol) was evaporated out. The composite was labeled as QTi. Bare $\mathrm{TiO}_{2}$ was also used as a standard for comparison purposes. Before usage, all the samples were washed in distilled water at $60{ }^{\circ} \mathrm{C}$, dried at $110{ }^{\circ} \mathrm{C}$ overnight and kept in a desiccator.

\subsection{CHARACTERIZATION}

Nanotexture of both the carbon support and the titania/carbon composites was characterized by $\mathrm{N}_{2}$ (ASAP 2010, Micromeritics) and $\mathrm{CO}_{2}$ (Tristar 3000, Micromeritics) adsorption isotherms at -196 and $0{ }^{\circ} \mathrm{C}$, respectively. Before the experiments, the samples were outgassed under vacuum (ca. $10^{-3}$ torr) at $120^{\circ} \mathrm{C}$ overnight. The isotherms were used to calculate the specific surface area, $\mathrm{S}_{\mathrm{BET}}$, and pore volumes using the density functional theory (DFT) and the DR formulism.

The as-received carbon and the carbon/titania composites were further characterized by the point of zero charge $[6,7]$ and thermogravimetric analysis using a Setaram Labsys thermal analyzer. The instrument settings were as follows: heating rate $15^{\circ} \mathrm{C} \mathrm{min}^{-1}$ and a $\mathrm{N}_{2}$ atmosphere with $50 \mathrm{~mL}$ $\min ^{-1}$ flow rate.

\subsection{ADSORPTION AND DEGRADATION OF PHENOL}

Dark adsorption and phenol photolysis and photodegradation experiments were performed under the same experimental conditions, by means of kinetics studies from batch experiments at room temperature. Briefly, for adsorption in dark conditions about $1 \mathrm{~g} \mathrm{~L}^{-1}$ of adsorbent was placed in dark glass flasks containing $400 \mathrm{~mL}$ of phenol solution (in distilled non buffered water) of initial concentration $100 \mathrm{mg} \mathrm{L}^{-1}$ (solution $\mathrm{pH}$ ca. 6 units). The suspensions were stirred (100 rpm) and small aliquots of the solution $(\sim 1.5 \mathrm{~mL})$ were taken out at fixed time intervals to measure the 
evolution of the adsorbate concentration, using a UV spectrometer (Shimadzu 2501). The extracted samples were reintroduced in the flasks in order to avoid changes in the total volume of solution.

Photocatalytic reaction conditions were optimized concerning the initial phenol concentration, catalyst loading and the $\mathrm{TiO}_{2} / \mathrm{AC}$ ratio. Experiments were carried out in a photoreactor of $400 \mathrm{~mL}$ of aqueous solution. The UV irradiation source was provided by a high pressure mercury lamp $(125 \mathrm{~W})$, vertically suspended in a cylindrical, double-walled quartz jacket cooled by flowing water, immersed in the solution. The water cell was used to control the temperature during the experiments, preventing any overheating of the suspension due to the irradiation. At regular intervals, aliquots of the solution were extracted and analyzed by reverse-phase HPLC (Spherisorb $\mathrm{C} 18,125 \mathrm{~mm} \times 4 \mathrm{~mm}$ ), using methanol-water mixtures as mobile phase, and a photodiode array detector. The samples were previously filtered using regenerated cellulose filter having mean pore size of $0.45 \mu \mathrm{m}$.

\section{RESULTS AND DISCUSSION}

\section{TEXTURAL AND CHEMICAL CHARACTERIZATION OF THE MATERIALS}

An activated carbon was used as support of $\mathrm{TiO}_{2}(15 \mathrm{wt} . \%)$ and as catalyst in the photo-assisted degradation of phenol in aqueous solution. The role of the carbon support on the efficiency of phenol degradation was evaluated and discussed in terms of its textural and chemical features. For this purpose, the carbon-based catalysts along with fresh titanium oxide (P25) were characterized by means of gas adsorption (Table 1).

The $\mathrm{N}_{2}$ adsorption isotherm of the carbon support belongs to type I/IV in the BDDT classification [8], with a broad opening of the knee at low relative pressures, which is characteristic of a microporous material with micropores of wide sizes and a considerable development of mesoporosity. This was further confirmed by combining the information obtained from $\mathrm{CO}_{2}$ adsorption isotherm. The pore volume determined by $\mathrm{CO}_{2}$ data is almost twice lower than the corresponding volume of micropores "seen" by $\mathrm{N}_{2}$ (evaluated by DR method in both cases), confirming that the wide microporosity of this carbon accounts for a large fraction of the overall porosity (Table 1). As expected, the immobilization of titania in the carbon matrix partially blocked the porosity of the carbon support, although the composite still displays a porous character with a relatively large pore volume and surface area. Similar observations have been reported in the literature $[2,4]$. A deep insight reveals that the modification of the porosity affected mostly the microporosity determined by $\mathrm{N}_{2}$ adsorption data (ca. $16 \%$ fall), whereas the narrow microporosity determined by $\mathrm{CO}_{2}$ remained almost unaltered. This suggests that the titania did not enter in the inner microporosity of the carbon support during the impregnation, remaining on the outer surface and most accessible (large) pores. Consequently, the micropores of smaller sizes remained unblocked. 
As regards the surface chemistry, the values of the $\mathrm{pH}_{\mathrm{PZC}}$ revealed that the basic nature of the carbon support ( $\mathrm{pH}_{\mathrm{PZC}} 8.9$ units) was slightly modified after the incorporation of titania. The composite presents a more neutral value ( $\mathrm{pH}_{\mathrm{PZC}} 6.8$ units) which suggests the occurrence of interactions between the graphene layers of the carbon support and titania. Although due to the synthetic route followed in the preparation of the carbon/titania composite no chemical bonding is expected between the $\mathrm{TiO}_{2}$ and the carbon support, it seems that there exists a weak interaction (likely charge transfer). In fact, the $\mathrm{pH}$ of a suspension of the same amount of titania in water is around 3.9 units (far below the 6.8 units of the composite), supporting this premise.

\section{PHENOL ADSORPTION AND PHOTODEGRADATION}

The performance of the catalysts was evaluated by kinetic measurements under both dark and UV irradiation (Figure 1) conditions. Solution $\mathrm{pH}$ was around 6 units in all the experiments; under these conditions phenol molecule is mainly in a non-dissociated state $\left(\mathrm{pK}_{\mathrm{a}} \sim 9.1\right)$, for which interactions between phenol molecule and the catalysts are predominantly dispersive (non electrostatic).

In the case of pure titania, analysis of the species in solution confirmed that no phenol degradation occurs under dark conditions. Moreover, the amount of phenol adsorbed was very low (removal efficiency below $3 \%$ ), which was expected given the non-porous nature of the bare photocatalyst (Table 1). On the other hand, when UV irradiation is applied, phenol is largely eliminated from the solution, being the removal efficiency close to $80 \%$ after 6 hours of irradiation. Fitting of the kinetic data of phenol disappearance from solution upon UV irradiation of $\mathrm{P} 25$ photocatalyst to the pseudo-second order kinetics model [9] showed that the rate of phenol degradation proceeds rather slowly (Table 2). This trend was more remarkable in the early stages of the process, as inferred from the initial rate $\left(h_{o}\right)$ and $t_{1 / 2}$ (half-life time) parameters estimated according to the equations $h=k_{2} q_{t}^{2}[9,10]$ and $t_{1 / 2}=1 / k_{2} q_{t},[10]$ where $q_{t}$ is the total amount of phenol that disappeared from solution (either adsorbed and/or decomposed) after 6 hours.

Analysis of the solution composition during irradiation of P25 indicated that as phenol concentration in solution decreases, rising amounts of p-benzoquinone (BZ) and hydroquinone (HQ) were detected (Figure 2). BZ was the dominant intermediate at the earlier stages of the reaction, and it seemed to be decomposed as the irradiation proceeds at longer time (ca. from 3 hours). In the case of $\mathrm{HQ}$, smaller concentrations were detected although the proportion remained somewhat constant after 2 hours of irradiation. Since P25 is a non-porous material, degradation subproducts remain in the solution unless they are allowed to be further mineralized; thus they are necessarily detected in the solution.

Phenol removal efficiency at dark conditions on the carbon support Q is almost $80 \%$ after 6 hours, as opposed to $54 \%$ in the case of the composite (QTi). Such behaviour seems reasonable, taking into account that at dark conditions no degradation occurs and the removal is exclusively due to 
classical adsorption on the porosity of the catalyst. Hence, this is consistent with the textural characterization of the samples, where it was shown that the immobilization of titania brought about an important decrease in the porous features of carbon support (Table 1).

Besides the removal efficiency, the textural changes induced in the titania loaded composite also affected the rate of phenol disappearance under dark conditions (Table 2), particularly during the first stages, as inferred from the threefold decrease in the half-time parameter. The overall removal rate evaluated from the pseudo-second order equation decreased almost twice in the titania loaded composite.

On the other hand, both the rate and amount of phenol disappearance increased after UV irradiation, even in the case of the un-doped carbon support. In the case of QTi composite, the rate of phenol removal upon irradiation was almost three times faster than in dark conditions, being the reaction specially favoured at short times (half time decreased from over $60 \mathrm{~min}$ to less than 14 min). The removal efficiency (that in a porous catalyst encompass both adsorption and photodegradation) was also significantly enhanced with respect to dark adsorption, going up to $98 \%$ after 6 hours of exposure to UV light in the presence of sample QTi. Compared to P25, the rate and removal efficiency were also largely improved upon irradiation of the composite. Thus, it would seem that the immobilization of $\mathrm{TiO}_{2}$ on the porous carbon support boosts the photoactivity of pure titania.

The increase in the rate constant upon irradiation can be ascribed to the preferential adsorption and surface concentration of the pollutant onto the carbon porosity, followed by a spontaneous transfer from the support to titania surface, where it is more rapidly decomposed due to the large concentration gradient between the two solid phases. In such case, there seems to exist a synergistic effect in the composite due to the combination of the adsorption capacity of the carbon and the photoactivity of titanium oxide. Similar observations about the synergic effect of activated carbon as additive to titania in the photodegradation of organic pollutants have been described in the literature [11-14].

What is more interestingly inferred from this study is that the activated carbon used as support appears to modify the phenol photodegradation pathway, as supported by the identification and quantification of the intermediate products detected in the aqueous solution (Figure 2). Traces of HQ and pyrocathecol (PYC) were detected after UV irradiation of QTi sample. HQ had already been formed upon irradiation of P25 photocatalyst, although at a higher concentration (Figure 2A). Another important difference is that HQ was only detected during the first couple of hours during the irradiation of QTi, whereas in the case of bare titania its concentration remained somewhat constant up to 3 hours and then started to fall at a slow rate. The second intermediate product detected during the irradiation of P25 -BZ- was not detected in the solution at any time when sample QTi is irradiated. Nevertheless, this does not proof that it is not formed; as the composite exhibits large surface area and pore volumes (partially inherited from the porous carbon support), 
it is reasonable to expect that a fraction of the degradation compounds are retained (adsorbed) inside the pores. As above mentioned, in the case of $\mathrm{TiO}_{2}$ all degradation intermediates may be detected in the solution because this is a non-porous material.

Additionally, traces of a new intermediate (PYC) were quantified in the solution when the carbon/titania composite is used as photocatalyst. These results suggest that the presence of a porous carbon matrix plays a more complex role on phenol photodegradation mechanism through the formation of different intermediates. Thus, it seems that despite titania immobilization on the carbon is carried out by physical mixture, the weak interactions occurring between the carbon surface and titania (supported by the slight change in the $\mathrm{pH}_{\mathrm{PZC}}$ in the composite) are capable of altering the mechanism of phenol photodegradation. Evidences of the different phenol oxidation mechanisms which proceed through the formation of various intermediates (including PYC, HQ, or BZ) has already been proposed in the literature $[15,16]$.

The outstanding role of the activated carbon support on the modification of the degradation pathway in the presence of the activated carbon material was further confirmed by investigating the removal kinetics of the carbon support itself under dark and UV irradiation conditions.

The first remarkable difference is that none of the intermediates observed when using the composite or P25 as photocatalysts (neither PYC, HQ nor BZ) are now detected in the solution. In fact, no additional compounds (except phenol) where detected, although we cannot discard the formation of smaller degradation intermediates (such as short alkyl chain organic acids) that are not detected by reverse-phase HPLC in a standard C18 column. This finding may be most likely due to the large porosity of the carbon support (Table 1), so that phenol and/or any intermediate compound would be retained (adsorbed) in the porous network of the carbon support. What seems interesting to be mentioned is that phenol removal efficiency increased significantly, going from $76 \%$ in dark conditions up to $98 \%$ after UV exposure. Moreover, the rate of phenol disappearance is also largely accelerated after irradiation: the rate of phenol adsorption on the carbon support (given by $k_{2}$ constant under dark conditions) is four times lower than the rate of phenol degradation (estimated by $k_{2}$ constant under UV irradiation).

Thus it is evident that the as-received activated carbon -Q- presents a large self-photoactivity towards phenol decomposition, larger than that of P25. Based on the intrinsic photoactivity of titanium oxide and on the measured self-photoactivity of the carbon support, it seemed reasonable to expect larger removal efficiencies when irradiating the composite. However it was not the case and the photoactivity of the carbon support is larger than that of the carbon/titania composite (removal efficiency is similar but degradation rate is faster in the carbon support). The eventual cumulative or synergistic effect on the removal efficiency due to the photoactivity of titania and the carbon support does not seem to rule this system. On the contrary, the immobilization of titania counteracts the self-photoactivity of the carbon support under irradiation (antagonist effect). This is clearly observed at short reaction times (Figure 1). 
Such behaviour may be attributed to two factors: the fall in the porosity of the composite, and a blockage of the photoactive centers in the carbon support after immobilization of titania. It may happen that the titania covering the carbon surface in QTi sample prevents the UV irradiation from accessing the carbon photoactive sites, thus acting as inhibiting the carbon self-photoactivity.

Taking this in mind, the performance of the composite under UV irradiation could be explained in two ways: a synergistic effect of the carbon support that enhances the photoactivity of P25 towards or in terms of an inhibition of the self-activity of the activated carbon support caused by the immobilization of $\mathrm{TiO}_{2}$. In the latter case, the negative impact on the carbon self-photoactivity applying at short times (below 1.5 hours of irradiation), would be somehow neutralized at longer times (above 3 hours) likely by the photoactivity of the inorganic oxide. Although the synergic effect of carbon on the activity of titania is well documented in the literature [2,4,11], this antagonist effect has not been reported so far.

It should also be mentioned that a detailed analysis of the carbon chemical composition revealed a large ash content (ca. 11 wt.\%). Consequently, we cannot neglect an additional contribution of the mineral matter in the photodegradation of phenol. For this reason, a thorough analysis of the inorganic species that compose the carbon ash is being the subject of our current investigations.

The analysis of the textural features of the carbon materials after being submitted to dark adsorption and UV irradiation (dark and UV series in Table 1) provides an interesting viewpoint of the phenol disappearance process. As a general rule, there is a subterfuge trend in the sense that despite the removal efficiency after UV irradiation is much larger than in dark conditions, the textural properties of the exhausted materials do not follow the same logic.

The porosity of the carbon support after phenol removal under UV irradiation seems to be somewhat less blocked than that after dark adsorption. The surface area and pore volumes in Q (UV) sample are rather close than those of Q (dark) sample (small differences in the calculated values are within the limits of the error of the gas adsorption data). This is rather surprising taking into account that the removal efficiency in the former is 1.3 times larger than in the latter. It suggests that under UV irradiation, the degradation compounds that would be retained in the pores are either smaller molecules that cause a lower blockage of the porosity, or they are present at lower concentration -therefore the fall in the porosity is quantitatively lessened-.

At converse, the trend observed for the carbon/titania composite is different. In this case, again the values of the textural parameters are very close, although smaller for the sample which has been irradiated under UV light (Table 1). The removal efficiency of the composite is still larger under UV irradiation than in dark conditions; therefore this suggests that the degradation compounds are either present at higher concentration causing a more pronounced the blockage of the pores in QTi (UV) sample, or they are larger molecules (which could be due to an incomplete or less quantitative mineralization of phenol). 


\section{CONCLUSIONS}

In this study we have shown that nature of a porous carbon used as photocatalyst and support for immobilization of titania plays an outstanding role in the mechanism of phenol photodegradation. Compared to bare titania, new intermediate subproducts are detected in the presence of the carbon support (carbon/titania composite), confirming that the carbon matrix promotes the photodegradation of phenol through a different mechanism. The rate of the process is also largely accelerated in the presence of the carbon support; the performance largely depends on the textural and chemical features on the carbon.

Indeed, although titania immobilization on the carbon support is carried out by physical mixture, measurement of the $\mathrm{pH}_{\mathrm{PZC}}$ suggests the occurrence of weak interactions between the carbon surface and the titania, which provoke the enhancement in the photodegradation of phenol. The selected activated carbon support exhibits a large self-photoactivity, which is greatly suppressed by the immobilization of titania on the composite. In this case, besides the synergistic effect of the carbon over the photodegradation activity of titania, there is an antagonistic effect of the inorganic oxide over the photoactivity of the carbon support itself.

\section{ACKNOWLEDGMENTS}

This work was supported by the Spanish MICINN (CTM2008-01956). COA thanks the Spanish MEC for a Ramon y Cajal Research Contract. LFV thanks CSIC for a predoctoral fellowship.

\section{REFERENCES}

[1] H. Choi, E. Stathatos, D. Dionysiou, Sol-gel preparation of mesoporous photocatalytic $\mathrm{TiO}_{2}$ films and $\mathrm{TiO}_{2} / \mathrm{Al}_{2} \mathrm{O}_{3}$ composite membranes for environmental applications. Appl. Catal. B 63 (2006) 60-67.

[2] B. Tryba, A.W. Morwski, M. Inagaki, Application of $\mathrm{TiO}_{2}$-mounted activated carbon to the removal of phenol from water, Appl. Catal. B 41 (2003) 427-433.

[3] C.G. Silva, W. Wang, J.L. Faria, Photocatalytic and photochemical degradation of mono-, di- and tri-azo dyes in aqueous solution under UV irradiation, J. Photochem. Photobiol. A: Chem. 181 (2006) 314-324.

[4] B. Tryba, Photocatalytic Activity of $\mathrm{TiO}_{2}$ by Carbon and Iron Modifications, Int. J. Photoenergy 721824 (2008) 1-15.

[5] X. Zhang X, M. Zhou, L. Lei, $\mathrm{TiO}_{2}$ photocatalyst deposition by MOCVD on activated carbon, Carbon 44 (2006) 325-333.

[6] J.S. Noh, J.A. Schwarz, J.A. Estimation of the point of zero charge of simple oxides by mass titration. J. Colloid Interf. Sci. 130 (1989) 157-164.

[7] C.O. Ania, B. Cabal, J.B. Parra, J.J. Pis, Importance of the Hydrophobic Character of Activated Carbons on the Removal of Naphthalene from the Aqueous Phase, Ads. Sci. Technol. 25 (2007) 155-168.

[8] B. Brunauer, L.S. Deming, W.E. Deming, E. Teller, On a theory of the van der Waals adsorption of gases. J. Am. Chem. Soc. 62 (1940) 1723-1732.

[9] Y.S. Ho, Review of second-order models for adsorption systems. J. Hazard. Mater. B136 (2006) 681-689.

[10] W.-T. Tsai, C.-W. Lai, T.-Y. Su, Adsorption of bisphenol-A from aqueous solution onto minerals and carbon adsorbents, J. Haz. Mat. B134 (2006) 169-175 
[11] J. Matos, J. Laine, J.M. Herrmann, Synergy effect in the photocatalytic degradation of phenol on a suspended mixture of titania and activated carbon, Appl. Catal. B 18 (1998) 281-291.

[12] Y. Ao, J. Xu, X. Shen, D. Fu, C. Yuan, Magnetically separable composite photocatalyst with enhanced photocatalytic activity, , J. Haz. Mat. 160 (2008) 295-300.

[13] T. Cordero, J.M. Chovelon, C. Duchamp, C. Ferronato, J. Matos, Surface nano-aggregation and photocatalytic activity of TiO2 on H-type activated carbons, Appl. Catal. B: Environ. 73 (2007) 227-235.

[14] S.X. Liu, X.Y. Chen, X. Chen, $\mathrm{A} \mathrm{TiO}_{2} / \mathrm{AC}$ composite photocatalyst with high activity and easy separation prepared by a hydrothermal method, , J. Haz. Mat. 143 (2007) 257-263.

[15] A. Santos, P. Yustos, A. Quintanilla, F. Garcia-Ochoa, Kinetic model of wet oxidation of phenol at basic pH using a copper catalyst, Chem. Eng. Sci., 60 (2005) 4866-4878.

[16] A. Santos, P. Yustos, A. Quintanilla, S. Rodríguez and F. Garcia-Ochoa, Route of the catalytic oxidation of phenol in aqueous phase, Appl. Catal. B: Environ. 39 (2002) 97-113.

Table 1. Textural parameters of the catalysts obtained from $\mathrm{N}_{2}$ and $\mathrm{CO}_{2}$ adsorption isotherms at -196 and $0{ }^{\circ} \mathrm{C}$, respectively. Textural parameters of the catalysts after phenol removal under dark conditions and UV irradiation are also compiled for comparison.

\begin{tabular}{lcccccc}
\hline & $\begin{array}{c}S_{\text {ВЕT }} \\
{\left[\mathrm{m}^{2} \mathrm{~g}^{-1}\right]}\end{array}$ & $\begin{array}{c}V_{\text {TOTAL }}{ }^{a} \\
{\left[\mathrm{~cm}^{3} \mathrm{~g}^{-1}\right]}\end{array}$ & $\begin{array}{c}V_{\text {MICRO }}{ }^{b} \\
{\left[\mathrm{~cm}^{3} \mathrm{~g}^{-1}\right]}\end{array}$ & $\begin{array}{c}V_{\text {MESO }}{ }^{b} \\
{\left[\mathrm{~cm}^{3} \mathrm{~g}^{-1}\right]}\end{array}$ & $\begin{array}{c}\mathrm{WO} \mathrm{N}_{2}{ }^{c} \\
{\left[\mathrm{~cm}^{3} \mathrm{~g}^{-1}\right]}\end{array}$ & $\begin{array}{c}W_{\mathrm{CO}}{ }^{c} \\
{\left[\mathrm{~cm}^{3} \mathrm{~g}^{-1}\right]}\end{array}$ \\
\hline P25 & 53 & 0.118 & - & - & - & - \\
Q & 1031 & 0.524 & 0.316 & 0.089 & 0.488 & 0.185 \\
QTi & 876 & 0.480 & 0.264 & 0.062 & 0.425 & 0.183 \\
\hline Q (dark) & 812 & 0.406 & 0.242 & 0.065 & 0.447 & 0.167 \\
QTi (dark) & 803 & 0.442 & 0.239 & 0.060 & 0.409 & 0.144 \\
\hline \multicolumn{7}{c}{ After phenol removal under UV irradiation } \\
\hline Q (UV) & 838 & 0.423 & 0.255 & 0.071 & 0.464 & 0.157 \\
QTi (UV) & 763 & 0.412 & 0.229 & 0.054 & 0.392 & 0.146 \\
\hline
\end{tabular}

a evaluated at relative pressures of 0.99

${ }^{\mathrm{b}}$ evaluated by DFT method applied to $\mathrm{N}_{2}$ adsorption data using slit-shaped pore model

c evaluated by DR approach

Table 2. Kinetic data of phenol disappearance (decomposition and/or adsorption) on the studied materials, obtained from fitting the experimental data to the pseudo-second order kinetic model: $k_{2}$ is the pseudosecond order rate constant; $h_{0}$ is the initial rate constant; $t_{1 / 2}$ is the half-life time and $r e$ is the experimental removal efficiency

\begin{tabular}{lccccc}
\hline & $\begin{array}{c}\boldsymbol{k}_{\mathbf{2}} \mathbf{1 0}^{\mathbf{1}} \\
\left(\mathbf{g ~ m}^{-1} \mathbf{m i n}^{-\mathbf{1}}\right)\end{array}$ & $\mathbf{r}^{\mathbf{2}}$ & $\begin{array}{c}\boldsymbol{h}_{\mathbf{0}} \\
\left(\mathbf{m g ~ g}^{-\mathbf{1}} \mathbf{m i n}^{-\mathbf{1}}\right)\end{array}$ & $\begin{array}{c}\boldsymbol{t} \mathbf{1} \mathbf{2} \\
(\mathbf{m i n})\end{array}$ & $\begin{array}{c}\boldsymbol{r e} \\
(\boldsymbol{\%})\end{array}$ \\
\hline P25 (dark) & - & - & - & - & 3 \\
Q (dark) & 5.38 & 0.999 & 0.0049 & 22.5 & 76 \\
QTi (dark) & 2.47 & 0.991 & 0.0019 & 62.3 & 54 \\
\hline P25 (UV) & 0.72 & 0.987 & 0.0007 & 128.9 & 78 \\
Q (UV) & 23.9 & 0.999 & 0.023 & 4.2 & 98 \\
QTi (UV) & 6.86 & 0.999 & 0.007 & 13.6 & 98 \\
\hline
\end{tabular}




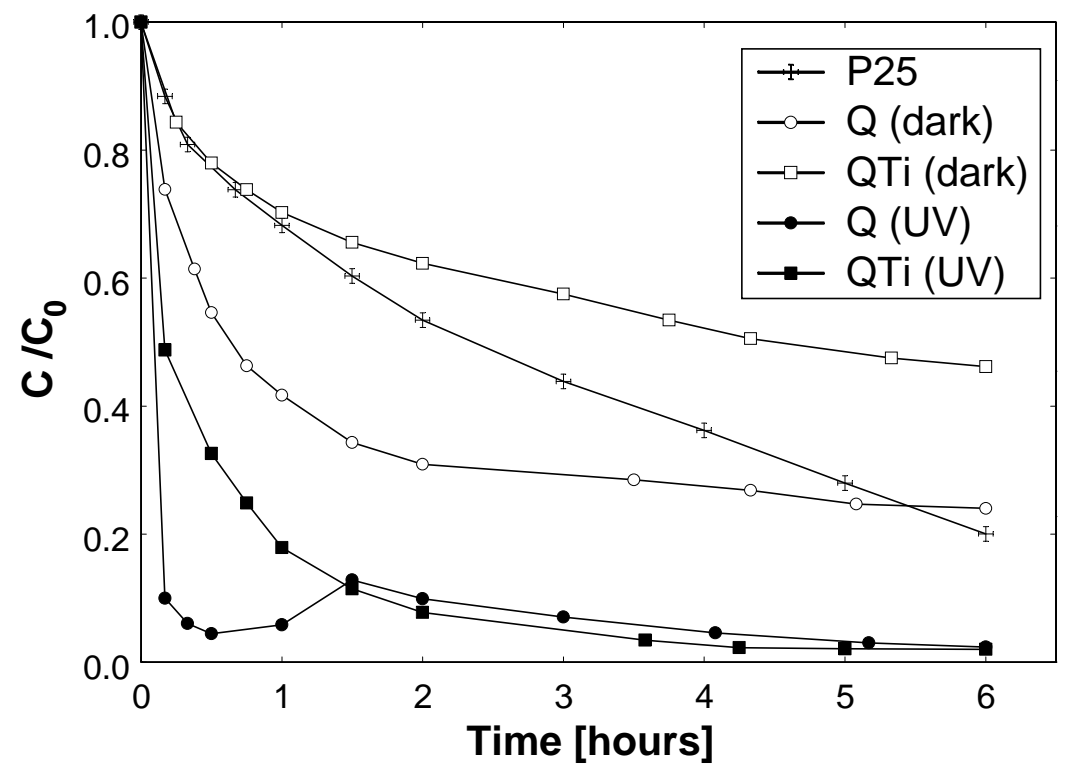

Figure 1. Phenol concentration decay curves on the investigated catalysts under dark adsorption and UV irradiation.
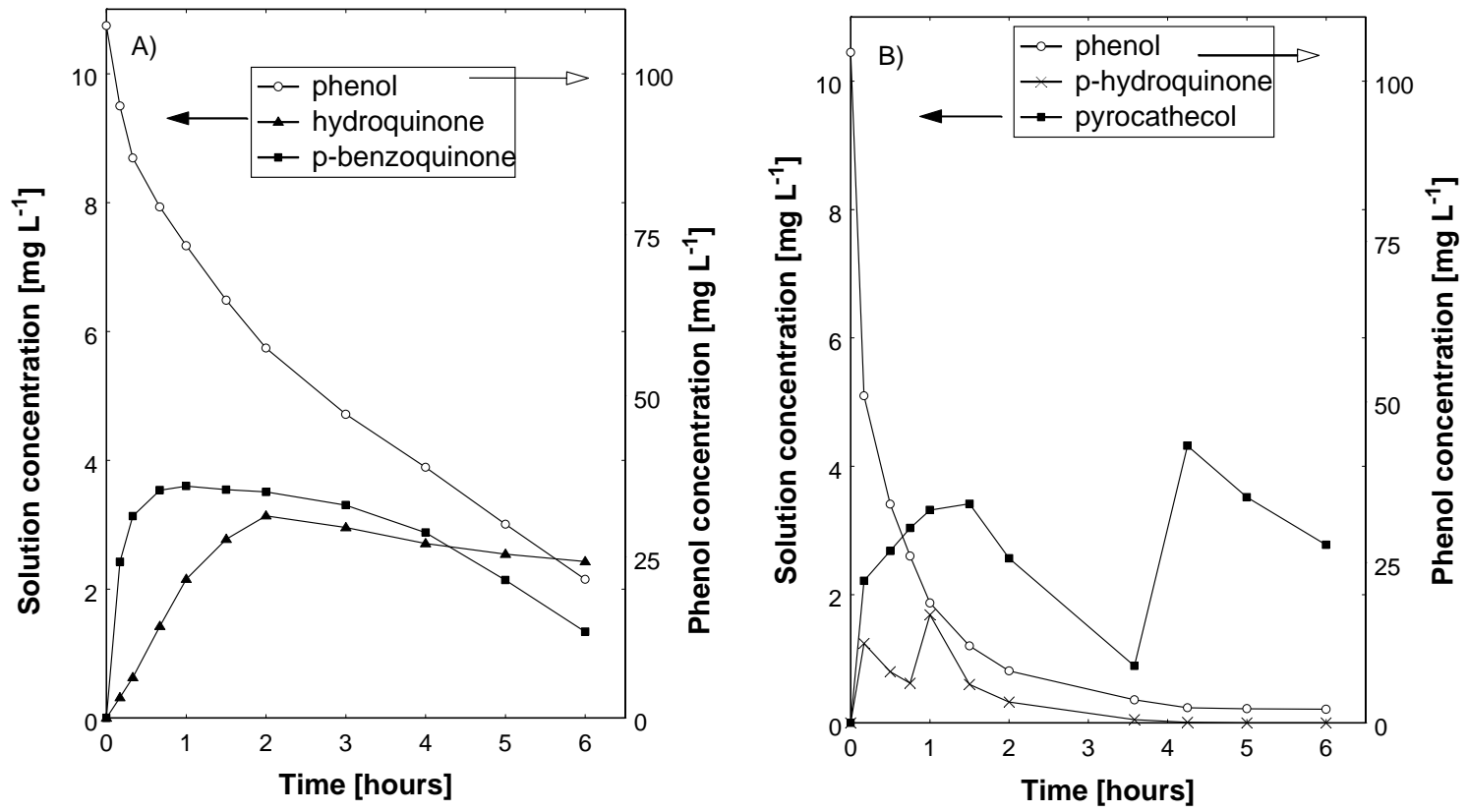

Figure 2. Evolution of phenol decomposition intermediates upon UV irradiation on the different materials investigated. A) commercial titania and B) carbon/titania composite. Concentrations of the degradation intermediates are read on the left axis, whilst phenol concentrations are read on the right $\mathrm{Y}$ axis. 